\title{
A case of recurrent, spontaneous rhabdomyolysis: A manifestation of McArdle disease
}

\author{
Felistia Nicole Crowder ${ }^{1}$, Mohamed Nasser ${ }^{2}$, Jenna Noelle Sizemore*3 \\ ${ }^{1}$ West Virginia University School of Medicine, West Virginia, United States \\ ${ }^{2}$ Department of Neurology, West Virginia University School of Medicine, West Virginia, United States \\ ${ }^{3}$ Department of Internal Medicine, West Virginia University School of Medicine, West Virginia, United States
}

Received: November 11, 2019

DOI: $10.5430 /$ crim.v7n1p11
Accepted: January 12, 2020

Online Published: February 17, 2020

\begin{abstract}
Background: McArdle Disease is a glycogen storage disease caused by an inherited deficiency in myophosphorylase, an enzyme that initiates the breakdown of muscle glycogen for glycolysis. Due to clinical variability and low prevalence, this disease is often delayed in diagnosis. Patients may present in early adulthood, suffering from exercise intolerance, muscle fatigue, myalgias, and potential end-organ damage from rhabdomyolysis and myoglobinuria. Diagnosis is confirmed with muscle biopsy showing an absence of myophosphorylase activity and can be confirmed with PYGM genetic sequencing. Treatment is preventative through dietary control with the use of complex carbohydrates and the ingestion of simple carbohydrates prior to aerobic activity.

Case presentation: A 19-year-old Caucasian male presented with a four-day history of rhabdomyolysis and acute kidney injury triggered by diving into cold water and swimming. The patient had developed muscle spasms and dyspnea, followed by a period of emesis, dysuria, and numbness in the lower extremities. Laboratory tests revealed creatinine kinase levels greater than 100,000 $\mathrm{U} / \mathrm{L}$ and creatinine of $1.6 \mathrm{mg} / \mathrm{dl}$. The patient had experienced a similar episode three months prior that occurred after sprinting, requiring hospitalization secondary to rhabdomyolysis. After eliminating reversible causes for rhabdomyolysis, there was high clinical suspicion for McArdle Disease, confirmed by muscle biopsy with absent phosphorylase activity. The patient was managed with parenteral fluids to treat the rhabdomyolysis and counseled on preventative dietary and exercise strategies.

Conclusions: The late onset of presentation of McArdle Disease should prompt consideration of metabolic myopathies in a systemic evaluation for cases of recurrent rhabdomyolysis.
\end{abstract}

Key Words: McArdle Disease, Myophosphorylase deficiency, Glycogen storage disease, Rhabdomyolysis

\section{INTRODUCTION}

Rhabdomyolysis is a potentially life-threatening disease involving rapid dissolution of damaged skeletal muscle following an insult, ${ }^{[1]}$ with subsequent leakage of toxic contents into the bloodstream. The evaluation of rhabdomyolysis utilizes a broad differential based on recurrent versus single episodes. McArdle Disease is a type of glycogen storage disease (Type V) characterized by a deficiency in the enzyme myophosphorylase, the form of glycogen phosphorylase found in skeletal muscle that breaks down muscle glycogen for glycolysis. This disease is most often inherited in an autosomal recessive pattern due to mutations in the PYGM gene. ${ }^{[2]}$ Although manifestations of the disease typically present in childhood as some form of exercise intolerance, time of onset and disease severity can vary among individuals based on factors such as baseline activity level,

\footnotetext{
*Correspondence: Jenna Noelle Sizemore; Email: jsizemo3@ hsc.wvu.edu; Address: PO Box 9160, Department of Medicine, Section of General Internal Medicine, 4th Floor HSCN, Suite 4086, Room 4087B, Morgantown, WV 26506, United States.
} 
diet, sex, and certain genotypes. ${ }^{[3]}$ Typical clinical features include exercise intolerance resulting in muscle crises, muscle weakness or spasms, elevated creatinine kinase levels, and the second wind phenomenon. ${ }^{[2,3]}$ Diagnosis can be made by the absence of myophosphorylase activity on muscle biopsy, and management focuses on prevention with diet and exercise. ${ }^{[3]}$

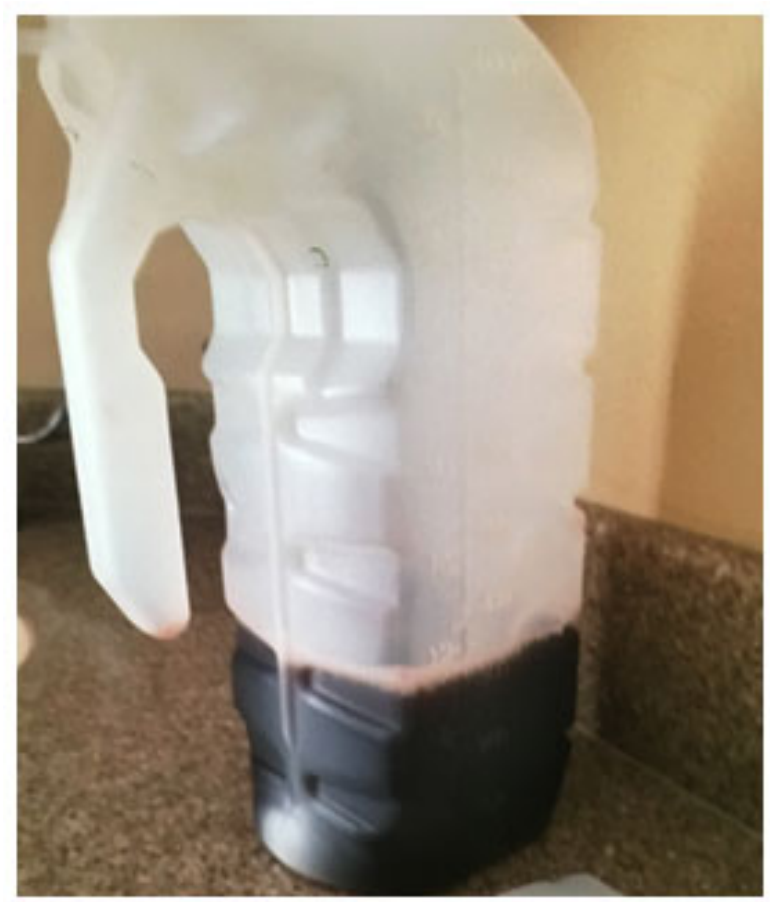

Figure 1. Urine sample showing myoglobinuria secondary to rhabdomyolysis

\section{Case Presentation}

\subsection{History}

A 19-year-old Caucasian male with past medical history of Attention Deficit Hyperactivity Disorder presented from a critical access hospital with a four-day history of rhabdomyolysis and acute kidney injury. The patient had gone swimming when he suddenly developed diffuse muscle spasms and difficulty breathing immediately upon diving into the water, prompting a water rescue. Following the episode, the patient had a prolonged period of non-bloody emesis, difficulty urinating, and numbness in his legs. Initial laboratory investigation at the outside hospital revealed an elevated creatinine kinase (CK) level greater than 100,000 U/L, abnormal renal function with creatinine $1.6 \mathrm{mg} / \mathrm{dl}$, and transaminitis The patient was treated with parenteral fluids while CK levels were monitored before being transferred to our facility for further work-up and management. The patient reported a similar episode three months prior when he developed muscle spasms after sprinting, with associated stiffness, dyspnea, emesis, and darkened urine that also required hospitalization secondary to rhabdomyolysis. Throughout his past, the patient had remained an active young male with performance on several athletic teams without significant myalgias or weakness during participation. Review of systems noted an unintentional weight loss over several months. Family history was significant for Charcot-Marie-Tooth in his sister and father, with onset of similar symptoms in his father at the same age as the patient.

\subsection{Investigations}

Investigation for acquired and reversible causes of rhabdomyolysis was conducted, including evaluation for infection, toxins, electrolyte imbalance, autoimmune disease, and thyroid disease. Urinalysis was positive for moderate blood with zero microscopic red blood cells, confirming myoglobinuria. Figure 1 is a photograph of the patient's urine upon initial presentation. Laboratory investigation revealed an elevation in aspartate aminotransferase (AST) to 2,101 U/L and alanine aminotransferase (ALT) to 1,043 U/L, which rapidly improved with aggressive hydration. Creatinine kinase (CK) was elevated at 259,000 U/L. Right upper quadrant ultrasonography was unremarkable. Both aldolase and lactate dehydrogenase levels were mildly elevated, which are nonspecific markers for muscular damage. A laboratory investigation for infectious causes, including viral serologies for Human Immunodeficiency Virus, Hepatitis A, Hepatitis B, Hepatitis C, and Epstein Barr Virus, was ultimately negative. Erythrocyte sedimentation rate (ESR) and C-reactive protein (CRP) levels were within normal limits. A comprehensive urine drug screen and serum toxin screen were unremarkable, ruling out drug-induced etiologies. Electrolytes were within normal ranges, and creatinine had normalized with aggressive hydration. The immunological work-up was also negative, including tests for antinuclear antibodies (ANA) and anti-Jo-1 antibodies. Thyroid stimulating hormone (TSH) levels were within normal limits. Carnitine levels and fatty acid profile both returned within normal limits. Electromyography (EMG) testing was performed and revealed abnormalities suggesting an underlying myopathy with muscle membrane irritability in both upper and lower extremities, as well as active denervation in the upper extremities with myopathic motor units. Nerve conduction studies showed no evidence of demyelinating neuropathy. Results of a left biceps muscle biopsy revealed primary myopathy with phosphorylasenegative histochemistry confirming the diagnosis of McArdle Disease. The patient and family declined genetic testing, citing financial concerns. With aggressive hydration, CK levels declined to normal limits within six days of initial insult. Table 1 depicts the downward progression of CK levels during the hospital admission. Following diagnosis, the patient was 
counseled on maintaining a protein-rich diet and ingesting simple carbohydrates before activity and was given a prescription for targeted physical therapy designed for patients with metabolic myopathies to help maintain an active quality of life.

Table 1. CK Levels throughout Hospitalization

\begin{tabular}{ll}
\hline Date/Time & CK Level \\
\hline Day $1-9: 24 \mathrm{am}$ & 259,000 \\
Day $1.1-2: 59 \mathrm{pm}$ & 155,000 \\
Day $1.2-7: 20 \mathrm{pm}$ & 126,000 \\
Day $2.1-1: 23 \mathrm{am}$ & 81,000 \\
Day 2.2 - 6:39am & 60,618 \\
Day 3 - 5:09am & 18,428 \\
Day 4-5:51am & 7,671 \\
Day 5 - 5:26am & 3,863 \\
\hline
\end{tabular}

\section{Discussion}

McArdle Disease is a glycogen storage disorder of myophosphorylase deficiency that is inherited in an autosomal recessive fashion. The most common mutations are found at the PYGM gene, with more than 100 mutations associated with McArdle Disease. ${ }^{[4]}$ Myophosphorylase is the form of glycogen phosphorylase found in skeletal muscle that converts stored glycogen into glucose-1-phosphate, which then enters glycolysis to produce available energy. ${ }^{[5]}$ In the absence of myophosphorylase, the body is unable to use the glycogen stored in skeletal muscle for energy. This disease is known as a pure myopathy since only the muscle isoform of glycogen phosphorylase is affected while the liver and brain isoforms remain unaffected. ${ }^{[2,5]}$ Although patients with McArdle Disease are unable to utilize the stored muscle glycogen as energy, the ability to use other sources to fuel activity remains intact. Therefore, ingestion of simple carbohydrates before activity is known to improve exercise tolerance in these patients. ${ }^{[3,5,6]}$ The hallmark clinical manifestation of McArdle Disease is exercise intolerance. This often presents in the form of muscle crises after strenuous activity. ${ }^{[7]}$ Symptoms may include muscle fatigue and myalgias, with the possibility of progression to rhabdomyolysis due to severe muscle damage and leakage of muscle proteins. ${ }^{[2,3,8]}$ Clinical suspicion is based on the following elements: exercise intolerance, chronic muscle weakness, increased CK at rest unprovoked by activity, one or more previous episodes of rhabdomyolysis, and the second wind phenomenon. ${ }^{[3]}$ The second wind phenomenon is considered pathognomonic for McArdle Disease and involves the ability to return to activity after a brief rest at the onset of muscle fatigue, which is thought to be facilitated by an increase in blood flow and delivery of free fatty acids, with an increase

Published by Sciedu Press in fatty acid metabolism and glucose utilization from the blood. ${ }^{[9-11]}$ Therefore, exercise tolerance is enhanced by allowing the body to use other sources for energy to fuel the activity. As clinical symptoms can vary in severity, diagnosis is often delayed. ${ }^{[12]}$ Elevation in baseline CK may prompt consideration for metabolic myopathies and is a common reason for prompting further investigation. ${ }^{[13]}$ The diagnosis can be confirmed by muscle biopsy, with samples often taken from the vastus lateralis or biceps brachialis, in order to look for the presence or absence of myophosphorylase activity. ${ }^{[2,3]}$ Biopsy results typically include decreased levels of myophosphorylase and increased glycogen on PAS stain. ${ }^{[3,14,15]}$ Genetic testing for PYGM mutations can be used to confirm the diagnosis. ${ }^{[2,3]}$ Although not routinely done today, traditionally an ischemic forearm test would be performed revealing no elevations in lactic acid. ${ }^{[3,14,15]}$ Since many cases of McArdle Disease may present as rhabdomyolysis, it is important to keep a broad differential for the multiple causes of rhabdomyloysis. Investigation for the etiology of rhabdomyolysis should begin with a thorough patient and family history, recent history of trauma, medication use, and substance use disorders. ${ }^{[16-18]}$ Laboratory investigation should include electrolytes, toxicology screen, and thyroid function tests to search for acquired and treatable causes. ${ }^{[16-18]}$ After acquired pathologies have been eliminated, glycogen storage diseases, fatty acid metabolism disorders, mitochondrial myopathies, and structural muscle diseases should be considered on the differential diagnosis and evaluated. ${ }^{[16-18]}$ The treatment for McArdle Disease is primarily aimed at nutritional and physical support since there is no definitive treatment or cure for this condition. A traditional diet often fails at maintaining blood glucose as a fuel source as stored muscle glycogen cannot be utilized. ${ }^{[3,7]}$ A structured diet rich in complex carbohydrates and low in fats is recommended, along with ingestion of simple carbs before beginning any form of strenuous activity. ${ }^{[3,7,19]}$ Regular aerobic conditioning at moderate intensity is also highly recommended as this will improve exercise tolerance and decrease symptom severity. ${ }^{[2,3,7]}$ Resistance training and isometric exercises have historically been avoided due to fear of provoking rhabdomyolysis. ${ }^{[9]}$ However, some studies have shown that resistance training can be safe and beneficial if the correct training protocol is followed. ${ }^{[8,19]}$ Although McArdle Disease is the most common glycogen storage disorder, it remains a rare, diagnostic challenge, with an estimated prevalence of 1/100,000 patients in the United States. ${ }^{[2,4]}$ Several studies note that diagnosis is often delayed after the first presenting symptoms, which are often in childhood. ${ }^{[3,12,15]}$ The clinical variability in disease severity and presentation likely result in under diagnosis, therefore underestimating the true 
prevalence of disease. ${ }^{[12,15]}$ While some patients may have mild symptoms of fatigue, others may present with muscle crises and marked proximal muscle weakness, making the diagnosis more obvious. ${ }^{[3]}$ Females are typically found to have more severe disease presentation compared to males. ${ }^{[3]}$ Patients with sedentary lifestyles may not present with the typical exercise intolerance symptoms, and diets rich in car- bohydrates at baseline may mask disease symptoms for a long period of time. ${ }^{[3]}$ Given its variable presentation, a genetic myopathy should be considered in the correct clinical context to prompt appropriate clinical investigations.

\section{CONFlicts of INTEREST Disclosure}

The authors have declared no conflicts of interest.

\section{REFERENCES}

[1] Torres PA, Helmstetter JA, Kaye AM, et al. Rhabdomyolysis: pathogenesis, diagnosis, and treatment. Ochsner J. 2015; 15(1): 58-69.

[2] Nogales-Gadea G, Godfrey R, Santalla A, et al. Genes and exercise intolerance: insights from McArdle disease. Physiol. Genomics. 2016; 48(2): 93-100. PMid:26465709. https ://doi .org/10.115 2/physiolgenomics.00076. 2015

[3] Leite A, Oliveira N, Rocha M. McArdle disease: a case report and review. Int Med Case Rep J. 2012;5:1-4. PMid:23754915. https://doi.org/10.2147/IMCRJ.S28664

[4] De Castro M, Johnston J, Biesecker L. Determining the prevalence of McArdle disease from gene frequency by analysis of next-generation sequencing data. Genet Med. 2015;17:1002-1006. PMid:25741863. https://doi.org/10.1038/gim.2015.9

[5] Nogales-Gadea G, Santalla A, Ballester-Lopez A, et al. Exercise and Preexercise Nutrition as Treatment for McArdle Disease. Med Sci Sports Exerc. 2016; 48(4): 673-679. PMid:26559449. https : //doi.org/10.1249/MSS.0000000000000812

[6] Vissing J, Haller RG. The Effect of Oral Sucrose on Exercise Tolerance in Patients with McArdle's Disease. N Engl J Med. 2003;349(26):2503-2509. PMid:14695410. https ://doi .org/10 .1056/NEJMoa031836

[7] Lucia A, Nogales-Gadea G, Pérez M, et al. McArdle disease: What do neurologists need to know? Nat Clin Pract Neurol. 2008; 4(10): 568-77. PMid:18833216. https://doi .org/10.1038/ncpneuro 0913

[8] Santalla A, Nogales-Gadea G, Ørtenblad N, et al. McArdle Disease: A Unique Study Model in Sports Medicine. Sports Med. 2014; 44(11): 1531-1544. PMid:25028051. https ://doi .org/10.100 7/s40279-014-0223-5

[9] Pietrusz A, Scalco RS, Quinlivan R. Resistance Exercise Training in McArdle Disease: Myth or Reality? Case Rep Neurol Med. 2018; 2018: 9658251 . Published online 2018 Sep. 30 . PMid:30363996. https://doi.org/10.1155/2018/9658251

[10] Haller G, Vissing J. Spontaneous "Second Wind" and GlucoseInduced Second "Second Wind" in McArdle Disease. Arch Neurol.
2002; 59: 1395-1402. PMid:12223025. https://doi.org/10.1 001/archneur.59.9.1395

[11] Vissing J, Haller RG. A diagnostic cycle test for McArdle's disease. Ann Neurol. 2003; 54: 539-542. PMid:14520671. https : //doi.org/10.1002/ana.10725

[12] Scalco RS, Morrow JM, Booth S, et al. Misdiagnosis is an important factor for diagnostic delay in McArdle disease. Neuromuscul Disord. 2017; 27: 852-855. PMid:28629675. https://doi.org/10.101 $6 / j . n m d .2017 .04 .013$

[13] Morrondo CD, Zarza LP, Tejado BSM. McArdle Disease: 2 Case Reports. Reumatol Clin. 2016; 12: 161-163. https ://doi.org/10 $.1016 /$ j.reumae .2015 .06 .004

[14] Miteff F, Potter HC, Allen J, et al. Clinical and laboratory features of patients with myophosphorylase deficiency (McArdle disease). J Clin Neurosci. 2011; 18(8): 1055-1058. PMid:21658951. https://doi.org/10.1016/j.jocn.2010.12.033

[15] Livingstone C, Riyami SA, Wilkins P, et al. McArdle's disease diagnosed following statin-induced myositis. Association of Clinical Biochemists. 2004;41:338-340. PMid:15298748. https : //doi .or g/10.1258/0004563041201554

[16] Chawla J. Stepwise approach to myopathy in systemic disease. Front Neurol [Internet]. 2011 Aug 5;2:49. Available from: https:// www.ncbi.nlm.nih.gov/pmc/articles/PMC3153853/https : //doi.org/10.3389/fneur.2011.00049

[17] Keltz E, Khan FY, Mann G. Rhabdomyolysis. The role of diagnostic and prognostic factors. Muscles Ligaments Tendons J [Internet]. 2014 Feb 24; 3(4): 303-12. Available from: https : //www.ncbi.n lm.nih.gov/pmc/articles/PMC3940504/

[18] Nance JR, Mammen AL. Diagnostic evaluation of rhabdomyolysis. Muscle Nerve. 2015; 51(6): 793-810. PMid:25678154. https : //doi.org/10.1002/mus. 24606

[19] Maté-Muñoz JL, Moran M, Pérez M, et al. Favorable Responses to Acute and Chronic Exercise in McArdle Patients. Clin J Sport Med. 2007;17:297-303. PMid:17620784. https://doi.org/10.1097/ JSM. Ob013e3180f6168c 